\title{
LIPOMA GAUSING A POSTERIOR INTEROSSEOUS NERVE LESION
}

\section{David A. Richmond, Burnley, England}

A man aged sixty-two years complained of inability to extend his left index finger and thumb. This paralysis had been progressing for about twelve weeks, and had been accompanied by pain in the upper arm radiating into the forearm. On examination, there was paralysis of the extensor muscles of the left thumb and index finger. There were no sensory changes. In the extensor muscles, just distal to their origin from the humerus, there was an ill defined intramuscular tumour.

Differential diagnosis-Since the patient was a painter, lead poisoning was considered, but was excluded. Because of the pain in the upper arm a cervical disc prolapse was suspected, and a radiograph showed marked narrowing of the disc space at the level of C.5-6. But there was no evidence that the paralysis was due to root pressure. A posterior interosseous

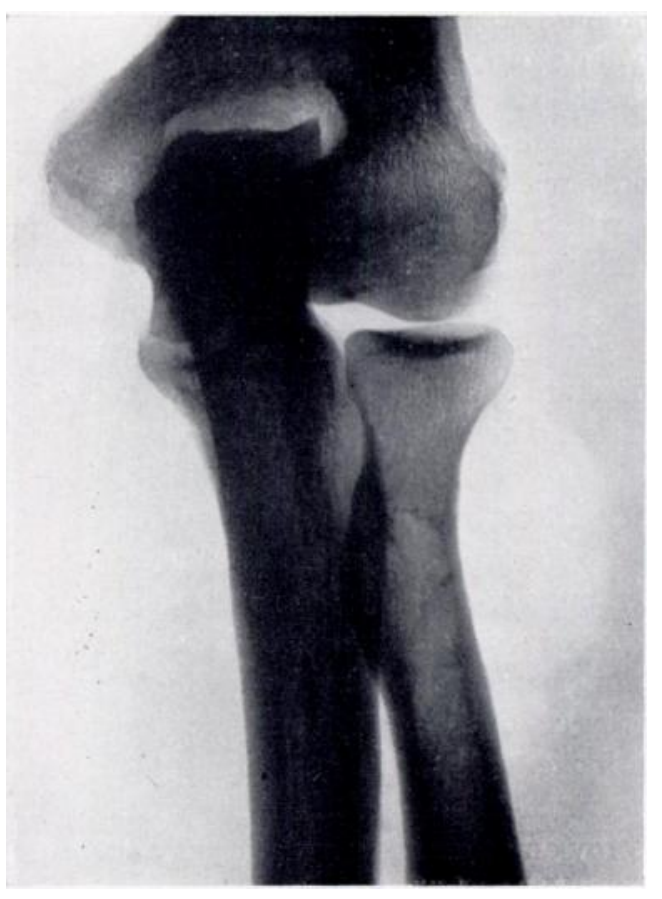

FIG. 1

Radiograph showing radiotranslucent area around neck of radius. nerve lesion seemed the correct diagnosis; the cause was assumed to be the intramuscular tumour near the extensor origin. Radiographs of the elbow showed a radiotranslucent, lobulated tumour lying lateral to the neck of the radius, and presenting the unusual appearance of an air bubble in the tissues (Fig. 1). Since the paralysis was gradually extending to involve all the finger extensors, operation was undertaken.

Operation-The radial nerve was exposed at the point of its division at the elbow. The tumour was found to be a lipoma lying under this point of division, in the substance of the supinator. The posterior interosseous nerve was stretched over the convexity of the tumour. The lipoma lay in contact with the periosteum covering the neck of the radius, and extended round the bone as far as its posterior aspect. It was removed without damage to the nerve.

After operation slow recovery occurred. Six months after operation the extensor digitorum was contracting against gravity, and after eleven months there was a flicker of movement in the extensor pollicis longus.

The disability was slight and the patient was back at work. When seen five months later all muscles had recovered.

Comment-This case is of interest because of the seeming rarity of peripheral nerve lesions caused by lipomata. In a search of the literature the author has failed to find a report of a similar case. 